\title{
QUARTIC NORMAL EXTENSIONS OF THE RATIONAL FIELD
}

\author{
TANG JIAN-ER
}

(Received 8 January 1990; revised 7 May 1990)

Communicated by J. H. Loxton

\begin{abstract}
There are two types of quartic normal extensions of the rational field, depending on the Galois group of the generating equation. All such extensions are described here in a uniquely parametrized form.

1991 Mathematics subject classification (Amer. Math. Soc.) 11 R 16.
\end{abstract}

It is well known that every quadratic extension of the rational field $Q$ is normal. This is no longer true for quadratic extensions of a quadratic field, for example $Q(\sqrt[4]{2})$ is not normal over $\mathbb{Q}(\sqrt{2})$. Quadratic extensions are easy to describe: as $D$ runs through all squarefree integers not equal to 1 , $\mathbb{Q}(\sqrt{D})$ runs through all quadratic (normal) fields. In the following we shall describe all normal quartic fields.

In a sense the normal quartic extensions of $\mathbb{Q}$ are well known. Indeed the only transitive permutation groups of order 4 are the cyclic group

$$
G_{1}=\{I,(1234),(13)(24),(1432)\}
$$

and the Klein group

$$
G_{2}=\{I,(12)(34),(13)(24),(14)(23)\}
$$

and so adjunction of a root of a quartic equation to $\mathbb{Q}$ generates a normal extension only if the Galois group of the equation over $\mathbb{Q}$ is either $G_{1}$ or $G_{2}$. From here it follows easily that a quartic normal extensions is either of the form $\mathbb{Q}(\sqrt{a+b \sqrt{D}})$ where $a, b$ are non-zero integers and $D$ is (C) 1991 Australian Mathematical Society 0263-6115/91 \$A2.00+0.00 
a squarefree greater than 1 , or of the form $\mathbb{Q}(\sqrt{A}, \sqrt{B})$ where $A, B$ are distinct squarefree integers not equal to 1 . Our purpose here is to find appropriate restrictions on these integers so as to obtain a unique description of the extensions.

TheOREM. Quartic normal extensions $K$ of the rational field $\mathbb{Q}$ are one of the following two types.

1. Let $D$ be a squarefree integer greater than 1 with no prime factor of the form $p \equiv-1(\bmod 4) ; r, s$, an integer solution of $r^{2}+s^{2}=D$ with $s>0$; and $k$ an odd squarefree integer such that $(k, D)=1$. Set $\alpha=D+s \sqrt{D}$. Then $K=\mathbb{Q}(\sqrt{k \alpha})$.

2. Let $A, B$ be squarefree integers not equal to 1 with $A<B$,

$$
\max (|A|,|B|)<|A B| /(A, B)^{2} \text {. }
$$

Then $K=\mathbb{Q}(\sqrt{A}, \sqrt{B})$.

The parameters $D, s, k$ in the first case and $A, B$ in the second case uniquely specify the extensions.

We need four lemmas.

LEMMA 1. Given the quartic equation

$$
x^{4}+a x^{3}+b x^{2}+c x+d=0
$$

over $\mathbb{Q}$, there exists a transformation $y=u+v x+w x^{2}, u, v, w \in \mathbb{Q}$, which transforms (1) into

$$
y^{4}+p y^{2}+q=0
$$

if and only if the Galois group of (1) over $\mathbb{Q}$ is a subgroup of the dihedral group

$$
G=\{I,(13),(24),(12)(34),(13)(24),(14)(23),(1234),(1432)\}
$$

where $I$ is the identity permutation.

Proof. Essentially this lemma is due to van der Ploeg [2], but not quite in such an explicit form and we give an independent proof. If there exist as transformation $y=u+v x+w x^{2}$ with rational coefficients, $u, v, w$ such that (1) can be changed into (2) then (1) is soluble by extraction of square roots alone, hence its group is a subgroup of $G$. Conversely suppose that the Galois group of (1) is a subgroup of $G$. Let the four roots of (1) be $x_{1}, x_{2}$, $x_{3}, x_{4}$, then $\psi=x_{1} x_{3}+x_{2} x_{4}$ is invariant under $G$ and so $\psi \in \mathbb{Q}$. Hence $\psi$ is a rational root of the Ferrari resolvent

$$
z^{3}-b z^{2}+(a c-4 d) z-a^{2} d+4 b d-c^{2}=0 .
$$


Let $\sigma_{1}, \sigma_{2}, \sigma_{3}$ denote the elementary symmetric polynomials of the roots of $(1)$; then

$$
\begin{aligned}
& \left(x_{1}-x_{2}+x_{3}-x_{4}\right)^{2}=\sigma_{1}^{2}-4 \sigma_{2}+4 \psi \\
& \quad\left(x_{1}-x_{2}+x_{3}-x_{4}\right)\left(x_{1}^{2}-x_{2}^{2}+x_{3}^{2}-x_{4}^{2}\right)=\sigma_{1}^{3}-4 \sigma_{1} \sigma_{2}+4 \sigma_{3}+2 \sigma_{1} \psi .
\end{aligned}
$$

Set $y_{i}=u+v x_{i}+w x_{i}^{2}, i=1,2,3,4$. Then the quartic equation with roots $y_{1}, y_{2}, y_{3}, y_{4}$ has the form (2) provided that $y_{1}+y_{2}+y_{3}+y_{4}=0$ and $y_{1}-y_{2}+y_{3}-y_{4}=0$, that is

$4 u+v \sigma_{1}+w\left(\sigma_{1}^{2}-2 \sigma_{2}\right)=0, v\left(x_{1}-x_{2}+x_{3}-x_{4}\right)+w\left(x_{1}^{2}-x_{2}^{2}+x_{3}^{2}-x_{4}^{2}\right)=0$.

Multiplying the second equation by $x_{1}-x_{2}+x_{3}-x_{4}$, we get

$$
v\left(\sigma_{1}^{2}-4 \sigma_{2}+4 \psi\right)+w\left(\sigma_{1}^{3}-4 \sigma_{1} \sigma_{2}+4 \sigma_{3}+2 \sigma_{1} \psi\right)=0
$$

giving the rational solution

$$
\begin{array}{r}
u=\frac{1}{2} a^{2} b+a c-2 b^{2}+\left(-\frac{1}{2} a^{2}+2 b\right) \psi, v=a^{3}-4 a b+4 c+2 a \psi, \\
w=a^{2}-4 b+4 \psi .
\end{array}
$$

It follows that (1) can be changed into (2) by the transformation $y=u+$ $v x+w x^{2}$.

\section{LEMMA 2. All integer solutions of}

$$
x^{2}+y^{2}=z^{4}
$$

are obtained by one of the following.

1. $x=k^{2}\left(u^{4}-6 u^{2} v^{2}+v^{4}\right), y=4 k^{2} u v\left(u^{2}-v^{2}\right), z=k\left(u^{2}+v^{2}\right)$ where $(u, v)=1, u+v \equiv 1(\bmod 2), k$ any integer,

2. $x=D\left(m^{2}-n^{2}\right), y=2 D m n, z=D l$, where $D>1$ is squarefree with no prime factor $\equiv-1(\bmod 4)$ and $m, n, l$ are integers satisfying $m^{2}+n^{2}=D l^{2}$.

This is essentially due to Euler, see [1, p. 621]. It can be obtained directly from the parametric solution of Pythagorean triples. Solutions of $m^{2}+n^{2}=$ $D l^{2}$ of course always exist.

Lemma 3. Let $D>1$ be squarefree with no prime factors of the form $p \equiv-1(\bmod 4), d \mid D, d>0$ with $d \equiv D(\bmod 2) ; r, s, t$ an integer solution of $r^{2}+s^{2}=D t^{2}$ with $(r, s)=1$. Then the equation $x^{2}+y^{2}=D$ has an integer solution $x, y$ such that $(r x+s y, s x-r y)=d$.

Proof. Since $(r, s)=1, t$ cannot be even or have a prime factor $p \equiv-1$ $(\bmod 4)$. Let $r+i s=(a+i b)(e+i f)(u+i v)^{2}$ be a factorization in the 
Gaussian domain such that $a^{2}+b^{2}=d, u^{2}+v^{2}=t,(u, v)=1$. This factorisation can clearly be accomplished so that no (odd) prime factor $p$ of $(D, t)$ divides $(e+i f)(u+i v)$. Set $x+i y=(a+i b)(e-i f)$, then $x^{2}+y^{2}=D$ and

$$
\begin{aligned}
(r+i s)(x-i y) & =r x+s y+i(s x-r y) \\
& =\left(a^{2}+b^{2}\right)(e+i f)^{2}(u+i v)^{2}=d(e+i f)^{2}(u+i v)^{2} .
\end{aligned}
$$

Since $(e+i f)(u+i v)$ has no rational integer divisor, $(r x+s y, s x-r y)=d$.

LEMMA 4. Let $D_{1}, D_{2}$ be squarefree integers greater than 1 with no prime factors $p \equiv-1(\bmod 4) ; r_{i}, s_{i}, t_{i}(i=1,2)$ integers satisfying $r_{i}^{2}+s_{i}^{2}=$ $D_{i} t_{i}^{2},\left(s_{i}, t_{i}\right)=1 ;$ and $k_{1}, k_{2}$ squarefree integers. Set

$$
\theta_{i}=\sqrt{k_{i}\left(t_{i} D_{i}+s_{i} \sqrt{D_{i}}\right)},
$$

$i=1,2$. Then $\mathbb{Q}\left(\theta_{1}\right)=\mathbb{Q}\left(\theta_{2}\right)$ if and only if $D_{1}=D_{2}$ and

$$
\frac{k_{2}\left(t_{1} t_{2} D+r_{1} r_{2}+\eta s_{1} s_{2}\right)}{2 k_{1} D}, \frac{k_{2}\left(t_{1} t_{2} D-r_{1} r_{2}-\eta s_{1} s_{2}\right)}{2 k_{1} D}
$$

are rational squares for $\eta=+1$ or -1 , where $D=D_{1}=D_{2}$.

Proof. Note that if either of the expressions (5) is a rational square then so is the other, since

$$
\begin{aligned}
\left(t_{1} t_{2} D+r_{1} r_{2}+\eta s_{1} s_{2}\right)\left(t_{1} t_{2} D-r_{1} r_{2}-\eta s_{1} s_{2}\right) & =t_{1}^{2} t_{2}^{2} D^{2}-\left(r_{1} r_{2}+\eta s_{1} s_{2}\right)^{2} \\
& =\left(r_{1} s_{2}-\eta r_{2} s_{1}\right)^{2} .
\end{aligned}
$$

Suppose that $D_{1} \neq D_{2}$, then $\sqrt{D_{1}} \in \mathbb{Q}\left(\theta_{1}\right)$ but $\sqrt{D_{1}} \notin \mathbb{Q}\left(\theta_{2}\right)$. Therefore if $\mathbb{Q}\left(\theta_{1}\right)=\mathbb{Q}\left(\theta_{2}\right)$ we must have $D_{1}=D_{2}=D$ and there exists $u, v, w$, $x \in \mathbb{Q}$ such that $\theta_{2}^{(j)}=u+v \theta_{1}^{(j)}+w \theta_{1}^{(j) 2}+x \theta_{1}^{(j) 3}, j=0,1,2,3$ where $\theta_{i}^{\prime}$, $\theta_{i}^{\prime \prime}, \theta_{i}^{\prime \prime \prime}$ are the conjugates of $\theta_{i}, i=1,2$. Since $\theta_{i}^{\prime \prime}=-\theta_{i}, \theta_{i}^{\prime \prime \prime}=-\theta_{i}^{\prime}$, we have $u=0, w=0$ hence

$$
\theta_{2}=v \theta_{1}+x \theta_{1}^{3}, \theta_{2}^{\prime}=v \theta_{1}^{\prime}+x \theta_{1}^{\prime 3}, \theta_{2}^{\prime \prime}=-v \theta_{1}-x \theta_{1}^{3}, \theta_{2}^{\prime \prime \prime}=-v \theta_{1}^{\prime}-x \theta_{1}^{\prime 3} \text {. }
$$

Consequently $\sigma_{2}=-v^{2}\left(\theta_{1}^{2}+\theta_{1}^{\prime 2}\right)-2 v x\left(\theta_{1}^{4}+\theta_{1}^{\prime 4}\right)-x^{2}\left(\theta_{1}^{6}+\theta_{1}^{\prime 6}\right)$,

$$
\sigma_{4}=\theta_{1}^{2} \theta_{1}^{\prime 2}\left(v^{2}+v x\left(\theta_{1}^{2}+\theta_{1}^{\prime 2}\right)+x^{2} \theta_{1}^{2} \theta_{1}^{\prime 2}\right)^{2}
$$

where $\sigma_{2}, \sigma_{4}$ are the elementary symmetric polynomials of the $\theta_{2}^{(j)}$. Hence $v$ and $x$ satisfy

$$
t_{1} v^{2}+2 k_{1}\left(2 t_{1}^{2} D-r_{1}^{2}\right) v x+k_{1}^{2}\left(4 t_{1}^{3} D^{2}-3 t_{1} r_{1}^{2} D\right) x^{2}=\frac{k_{2} t_{2}}{k_{1}}
$$


and

$$
v^{2}+2 k_{1} t_{1} D v x+k_{1}^{2} r_{1}^{2} D x^{2}=\xi \frac{k_{2} r_{2}}{k_{1} r_{1}}, \xi=+1 \text { or }-1 .
$$

Multiplying (6) by $\sqrt{D},(7)$ by $r_{1}$ and adding we obtain

$$
\left(t_{1} \sqrt{D}+r_{1}\right)\left(v+k_{1}\left(2 t_{1} D-r_{1} \sqrt{D}\right) x\right)^{2}=\frac{k_{2}}{k_{1}}\left(t_{2} \sqrt{D}+\xi r_{2}\right)
$$

hence

$$
v+k_{1}\left(2 t_{1} D-r_{1} \sqrt{D}\right) x= \pm \frac{1}{s_{1}} \sqrt{\frac{k_{2}}{k_{1}}\left(t_{2} \sqrt{D}+\xi r_{2}\right)\left(t_{1} \sqrt{D}-r_{1}\right)}
$$

for some sign on the right hand side.

Similarly subtracting $r_{1}$ times (7) from $\sqrt{D}$ times (6) we obtain

$$
v+k_{1}\left(2 t_{1} D+r_{1} \sqrt{D}\right) x= \pm \frac{1}{s_{1}} \sqrt{\frac{k_{2}}{k_{1}}\left(t_{2} \sqrt{D}-\xi r_{2}\right)\left(t_{1} \sqrt{D}+r_{1}\right)}
$$

for some sign on the right. Eliminating $v$ we have

$$
\begin{aligned}
2 k_{1} r_{1} s_{1} \sqrt{D} x= & \pm \sqrt{\frac{k_{2}}{k_{1}}\left(t_{2} \sqrt{D}-\xi r_{2}\right)\left(t_{1} \sqrt{D}+r_{1}\right)} \\
& \pm \sqrt{\frac{k_{2}}{k_{1}}\left(t_{2} \sqrt{D}+\xi r_{2}\right)\left(t_{1} \sqrt{D}-r_{1}\right)}
\end{aligned}
$$

for one of the four possible choices of sign on the right. Squaring gives

$$
\left(k_{1} r_{1} s_{1} x\right)^{2}=\frac{k_{2}}{2 k_{1} D}\left(t_{1} t_{2} D-\xi r_{1} r_{2}-\xi \eta s_{1} s_{2}\right) \text {. }
$$

Conversely suppose that for some $\eta= \pm 1$ it is true that $\left(k_{2} / 2 k_{1} D\right) \times$ $\left(t_{1} t_{2} D-\xi r_{1} r_{2}-\xi \eta s_{1} s_{2}\right)$ is a rational square for both $\xi=+1$ and $\xi=-1$. Then

$$
x=\frac{1}{k_{1} r_{1} s_{1}} \sqrt{\frac{k_{2}}{2 k_{1} D}\left(t_{1} t_{2} D-\xi r_{1} r_{2}-\xi \eta s_{1} s_{2}\right)}
$$

defines a rational number for $\xi= \pm 1$. Define $v$ by (8) with some sign on the right. Then $v$ and $x$ satisfy (6) and (7) as seen by reversing all calculations. But then $v$ is rational. For multiplying (7) by $t_{1}$ and subtracting from (6) we obtain

$$
v x=\frac{k_{2}}{2 k_{1}^{2} r_{1} s_{1}^{2}}\left(t_{2} r_{1}-\xi r_{2} t_{1}\right)-2 k_{1} t_{1} D x^{2}
$$

and rationality of $v$ follows. Hence $\theta_{2}=v \theta_{1}+x \theta_{1}^{3} \in \mathbb{Q}\left(\theta_{1}\right)$ and is easily seen to be of the form $\theta_{2}=\sqrt{k_{2}\left(t_{2} D+s_{2} \sqrt{D}\right)}$. 
We are now ready to prove our theorem. We first show that the extensions described in the theorem are indeed normal. In the case of the first type extension let $r^{2}+s^{2}=D, r>0, s>0, D>1$ squarefree with no prime factors $\pm-1(\bmod 4)$. Let $k$ be squarefree and set $\theta=\sqrt{k \alpha}, \alpha=D+s \sqrt{D}$. Its conjugates are

$$
\theta^{\prime}=\sqrt{k(D-s \sqrt{D})}=r\left(\theta^{2}-k D\right) / s \theta, \theta^{\prime \prime}=-\theta, \theta^{\prime \prime \prime}=-\theta^{\prime},
$$

therefore $\mathbb{Q}\left(\theta, \theta^{\prime}, \theta^{\prime \prime}, \theta^{\prime \prime \prime}\right)=\mathbb{Q}(\theta)$ is normal. In the case of the second type extension let $A, B$ be squarefree, not equal to $1, \theta=\sqrt{A}+\sqrt{B}$. Clearly, $\mathbb{Q}(\sqrt{A}, \sqrt{B})=\mathbb{Q}(\theta)$ is a quartic extension and the conjugates are

$$
\theta^{\prime}=\sqrt{A}-\sqrt{B}=(A-B) / \theta, \theta^{\prime \prime}=-\theta, \theta^{\prime \prime \prime}=-\theta^{\prime} \text {. }
$$

Therefore $\mathbb{Q}\left(\theta, \theta^{\prime}, \theta^{\prime \prime}, \theta^{\prime \prime \prime}\right)=\mathbb{Q}(\theta)$ is normal.

Now adjunction of a root of (1) to $\mathbb{Q}$ can generate a quartic normal extension only if the Galois group of the equation is either

$$
G_{1}=\{I,(1234),(13)(24),(1432)\}
$$

or

$$
G_{2}=\{I,(12)(34),(13)(24),(14)(23)\},
$$

over $\mathbb{Q}$. By Lemma 1 there exists a transformation $y=u+v x+w x^{2}$ over $\mathbb{Q}$ such that (1) is changed into (2). Therefore we may assume that our quartic normal field is generated by a root of an equation of the form (2). We may also assume that the coefficients $p, q$ in (2) are integers, otherwise multiply $y$ by a suitable integer to get rid of the denominator.

Suppose the group of (2) is $G_{1}$. We first show that the extension $K$ is of the following type:

$1^{*}$ Let $D$ be as in type $1, r, s, t$ an integer solution of $r^{2}+s^{2}=D t^{2}$ with $s>0, t>0,(s, t)=1$; and $k$ a squarefree integer. Then $K=\mathbb{Q}(\theta)$ where $\theta=\sqrt{k(t D+s \sqrt{D})}$. Let $\tau_{1}, \tau_{2}, \tau_{3}, \tau_{4}$ be the elementary symmetric polynomials of the roots $y_{1}, y_{2}, y_{3}, y_{4}$ of (2), then $\tau_{1}=0, \tau_{2}=p, \tau_{3}=0$, $\tau_{4}=q$, therefore

$\left(y_{1}+y_{2}-y_{3}-y_{4}\right)\left(y_{1}-y_{2}+y_{3}-y_{4}\right)\left(y_{1}-y_{2}-y_{3}+y_{4}\right)=\tau_{1}^{3}-4 \tau_{1} \tau_{2}+8 \tau_{3}=0$. The roots can be arranged so that $y_{1}-y_{2}+y_{3}-y_{4}=0$ say, and since $\tau_{1}=0$, we get

$$
y_{3}=-y_{1}, y_{4}=-y_{2}, y_{1}^{2}+y_{2}^{2}=-\tau_{2}=-p, y_{1}^{2} y_{2}^{2}=\tau_{4}=q .
$$

Consider $\psi=(1 / 16)\left(y_{1}+i y_{2}-y_{3}-i y_{4}\right)^{4}$ over $\mathbb{Q}(i)$. It belongs to $G_{1}$ and so its value is

$$
\begin{aligned}
\psi & =\left(y_{1}+i y_{2}\right)^{4}=\left(y_{1}^{2}+y_{2}^{2}\right)^{2}-8 y_{1}^{2} y_{2}^{2} \pm 4 i \sqrt{y_{1}^{2} y_{2}^{2}\left[\left(y_{1}^{2}+y_{2}^{2}\right)^{2}-4 y_{1}^{2} y_{2}^{2}\right]} \\
& =p^{2}-8 q \pm 4 i \sqrt{q\left(p^{2}-4 q\right)} \in \mathbb{Q}(i) .
\end{aligned}
$$


But $p$ and $q$ are integral, so there exists an integer $T$ such that $q\left(p^{2}-4 q\right)=$ $T^{2}$, hence $\left(p^{2}-8 q\right)^{2}+(4 T)^{2}=p^{4}$. By Lemma 2 , one of the following two conditions holds.

1. There exist integers $u, v$ with $(u, v)=1, u+v \equiv 1(\bmod 2)$, and an integer $k \neq 0$ such that $p=k\left(u^{2}+v^{2}\right)$ and

$$
p^{2}-8 q=k^{2}\left(u^{4}-6 u^{2} v^{2}+v^{4}\right) \text { or } 4 k^{2} u v\left(u^{2}-v^{2}\right) \text {. }
$$

2. There exists a squarefree integer $D>1$ with no prime factor $\equiv-1$ $(\bmod 4)$, and integers $m, n, l$ satisfying $m^{2}+n^{2}=D l^{2}$, such that $p=D l$ and $p^{2}-8 q=D\left(m^{2}-n^{2}\right)$ or $2 D m n$. In Case 1 we have $q=k^{2} u^{2} v^{2}$ or $(1 / 8) k^{2}\left(u^{2}-2 u v-v^{2}\right)^{2}$. If $(2)$ is

$$
y^{4}+k\left(u^{2}+v^{2}\right) y^{2}+k^{2} u^{2} v^{2}=0 \text { then }\left(y^{2}+k u^{2}\right)\left(y^{2}+k v^{2}\right)=0,
$$

and the equation is reducible and does not generate a quartic field. So (2) is

$$
y^{4}+k\left(u^{2}+v^{2}\right) y^{2}+\frac{1}{8} k^{2}\left(u^{2}-2 u v-v^{2}\right)^{2}=0,
$$

its roots are

$$
y=( \pm 1 / 2) \sqrt{-k\left[2\left(u^{2}+v^{2}\right) \pm \sqrt{2}\left(u^{2}+2 u v-v^{2}\right)\right]}
$$

with independent \pm signs. By definition $u^{2}+v^{2}$ and $u^{2}+2 u v-v^{2}$ are coprime,

$$
\left(u^{2}+2 u v-v^{2}\right)^{2}+\left(u^{2}-2 u v-v^{2}\right)^{2}=2\left(u^{2}+v^{2}\right)^{2},
$$

and so $\mathbb{Q}(y)$ is an extension of type $1^{*}$ with $D=2, t=u^{2}+v^{2}, s=$ $\left|u^{2}+2 u v-v^{2}\right|$.

In Case 2 we have

$$
q=(1 / 4) D n^{2} \text { or }(1 / 8) D(m-n)^{2} .
$$

If (2) is $y^{4}+D l y^{2}+(1 / 4) D n^{2}=0$, its roots are

$$
y=( \pm 1 / 2)(\sqrt{-l D+n \sqrt{D}} \pm \sqrt{-l D-n \sqrt{D}})
$$

with $m^{2}+n^{2}=D l^{2}$, hence $\mathbb{Q}(y)$ is an extension of type $1^{*}$ with $t=$ $|l| /(l, n), s=|n| /(l, n), r=m /(l, n), k=(-l /|l|)(l, n)$. If $(2)$ is $y^{4}+$ $D l y^{2}+(1 / 8) D(m-n)^{2}=0$ and $D$ is odd then

$$
y=( \pm 1 / 2) \sqrt{-l(2 D) \pm(m+n) \sqrt{2 D}} .
$$

By the equality $(m+n)^{2}+(m-n)^{2}=2 D l^{2}, \mathbb{Q}(y)$ is an extension of type $1^{*}$ with $t=|l| /(l, m+n), s=|m+n| /(l, m+n), k=(-l /|l|)(l, m+n)$. 
Similarly if $D$ is even, since $(m \pm n)^{2} \mp 2 m m=D l^{2}, m+n$ and $m-n$ must be even,

$$
y= \pm \sqrt{-l(D / 2) \pm((m+n) / 2) \sqrt{D / 2}}
$$

and by the equality $((m+n) / 2)^{2}+((m-n) / 2)^{2}=(D / 2) l^{2}, \mathbb{Q}(y)$ is again an extension of type $1^{*}$ with $t=|l| /(l,(m+n) / 2), s=|m+n| / 2(l,(m+n) / 2)$, $k=(-l /|l|)(l,(m+n) / 2)$. It follows that if the group of (2) is $G_{1}$ then adjunction of its roots to $\mathbb{Q}$ generates an extension of type $1^{*}$.

Next we show that any extension of type $1^{*}$ is of type 1 . So suppose $r^{2}+s^{2}=D t^{2},(s, t)=1, k$ squarefree, $\theta=\sqrt{k(t D+s \sqrt{D})}$. If here $2 \mid k$, set $\beta=t D+r \sqrt{D}, \bar{\beta}=t D-r \sqrt{D}$, then $\theta=\sqrt{k \beta / 2}+\sqrt{k \bar{\beta} / 2}$ with $(t, r)=1$ (since $(t, s)=1$ ) and clearly $\mathbb{Q}(\theta)=\mathbb{Q}(\sqrt{k \beta / 2})$. So we may assume $2+k$. Let $(k, D)=d, k=k_{1} d$, then $\left(k_{1}, D\right)=1$ and $2 \nmid k_{1}$, $2 \nmid d$ hence $D / d+D \equiv 0(\bmod 2)$. By Lemma 3 , the equation $x^{2}+y^{2}=D$ has an integer solution $x, y$ such that $(r x+s y, s x-r y)=D / d$. Now

$$
(r x+s y)^{2}+(s x-r y)^{2}=\left(r^{2}+s^{2}\right)\left(x^{2}+y^{2}\right)=(t D)^{2}
$$

hence there exist integers $u, v$ with $(u, v)=1, u+v \equiv 1(\bmod 2)$ such that $r x+s y=(D / d)\left(u^{2}-v^{2}\right)$ or $(D / d)(2 u v), t D=(D / d)\left(u^{2}+v^{2}\right)$. If $r x+s y=(D / d)\left(u^{2}-v^{2}\right)$, set $\eta=\sqrt{k_{1}(D+y \sqrt{D})}$ and apply Lemma 4 with

$$
\theta_{1}=\eta\left(r_{1}=x, s_{1}=y, t_{1}=1\right)
$$

and

$$
\theta_{2}=\theta\left(r_{2}=r, s_{2}=s, t_{2}=t, k_{2}=k\right) \text {. }
$$

The expressions (5) in Lemma 4 and $u^{2}, v^{2}$ respectively hence by the Lemma, $\mathbb{Q}(\eta)=\mathbb{Q}(\theta)$. Similarly if $r x+s y=(D / d)(2 u v)$ then $s x-r y=$ $(D / d)\left(u^{2}-v^{2}\right)$ and setting $\eta=\sqrt{k_{1}(D+x \sqrt{D})}$ we can apply Lemma 4 with $\theta_{1}=\eta \quad\left(r_{1}=y, s_{1}=x, t_{1}=1\right), \theta_{2}=\theta$. The expressions in (5) are now $v^{2}, u^{2}$ respectively, and we again conclude that $\mathbb{Q}(\eta)=\mathbb{Q}(\theta)$. In either case the extension is of type 1 . (Since $\mathbb{Q}(\sqrt{k \alpha})=\mathbb{Q}(\sqrt{k \bar{\alpha}}), \bar{\alpha}=D-s \sqrt{D}$, we may assume at any rate $s>0$ ).

To show uniqueness of the parameters $s, k$, suppose that

$$
\begin{aligned}
& \eta_{1}=\sqrt{k_{1}\left(D+s_{1} \sqrt{D}\right)}, \\
& \eta_{2}=\sqrt{k_{2}\left(D+s_{2} \sqrt{D}\right)}, \mathbb{Q}\left(\eta_{1}\right)=\mathbb{Q}\left(\eta_{2}\right) .
\end{aligned}
$$

By Lemma 4 (and changing the sign of $s_{1}$ if necessary)

$$
\left(k_{2} / 2 k_{1} D\right)\left(D+r_{1} r_{2}+s_{1} s_{2}\right)
$$


is a square. Now $r_{1}^{2}+s_{1}^{2}=D, r_{2}^{2}+s_{2}^{2}=D$, hence

$$
\left(r_{1} r_{2}+s_{1} s_{2}\right)^{2}+\left(r_{1} s_{2}-r_{2} s_{1}\right)^{2}=D^{2} \text {. }
$$

Set $\left(r_{1} r_{2}+s_{1} s_{2}, D\right)=d$, then there exist integers $u, v$ satisfying $(u, v)=1$, $u+v \equiv 1(\bmod 2)$ such that $r_{1} r_{2}+s_{1} s_{2}=d\left(u^{2}-v^{2}\right)$ or $d(2 u v), D=$ $d\left(u^{2}+v^{2}\right)$. Suppose $r_{1} r_{2}+s_{1} s_{2}=d\left(u^{2}-v^{2}\right)$, then

$$
k_{2}\left(D+r_{1} r_{2}+s_{2} s_{2}\right) / 2 k_{1} D=k_{2} u^{2} / k_{1}\left(u^{2}+v^{2}\right)
$$

hence $k_{1} k_{2}\left(u^{2}+v^{2}\right)$ is a square. But $\left(k_{1}, D\right)=1,\left(k_{2}, D\right)=1$ and $k_{1}$, $k_{2}, D$ are squarefree therefore $k_{1}=k_{2}$ and $u^{2}+v^{2}=1, d=D$. But then $r_{1} r_{2}+s_{1} s_{2}=D, r_{1} s_{2}-s_{1} r_{2}=0$ which together with $r_{1}^{2}+s_{1}^{2}=D, r_{2}^{2}+s_{2}^{2}=D$ give $r_{1}=r_{2}, s_{1}=s_{2}$.

If $r_{1} r_{2}+s_{1} s_{2}=d(2 u v)$ then

$$
\frac{k_{2}}{2 k_{1} D}\left(D+r_{1} r_{2}+s_{1} s_{2}\right)=\frac{(u+v)^{2} k_{2}}{2 k_{1}\left(u^{2}+v^{2}\right)}, 2 k_{1} k_{2}\left(u^{2}+v^{2}\right) \text { is a square. }
$$

As before, it implies $k_{1}=k_{2}, u^{2}+v^{2}=2, D=2 d, r_{1} r_{2}+s_{1} s_{2}=2 d=D$, $r_{1} s_{2}-s_{1} r_{2}=0$ hence $r_{1}=r_{2}, s_{1}=s_{2}$.

Finally suppose that the group of (2) is $G_{2}$. Then all three quantities $\psi_{1}=y_{1} y_{2}+y_{3} y_{4}, \psi_{2}=y_{1} y_{3}+y_{2} y_{4}, \psi_{3}=y_{1} y_{4}+y_{2} y_{3}$ belong to $G_{2}$ and are roots of the Ferrari resolvent of (2),

$$
z^{3}-p z^{2}-4 q z+4 p q=0
$$

Hence (9) has three rational roots. But (9) is $(z-p)\left(z^{2}-4 q\right)=0$ and so $q$ is a square $f^{2}$. Since $G_{2}$ is transitive, the roots

$$
y= \pm \frac{1}{2}(\sqrt{-p+2 f} \pm \sqrt{-p-2 f})
$$

of (2) are quartic algebraic numbers. Let

$$
-p+2 f=m^{2} M,-p-2 f=n^{2} N
$$

where $M, N$ are squarefree. Then the field is generated by any two of the squareroots of $M, N, M N /(M, N)^{2}$. Exactly one of these three numbers has a largest absolute value. Denoting by $A, B$ the other two we may assume $A<B, \max (|A|,|B|)<|A B| /(A, B)^{2}$ and we obtain $\mathbb{Q}(y)=\mathbb{Q}(\sqrt{A}, \sqrt{B})$, an extension of type 2 . Uniqueness of the parameters $A, B$ is obvious.

\section{Acknowledgement}

The author wishes to thank Professor G. Szekeres for his help in rewriting this paper. 


\section{References}

[1] L. E. Dickson, History of the theory of numbers, Vol. 2 (Chelsea, New York, 1952)

[2] C. E. van der Ploeg, 'Duality in non-normal quartic fields', Amer. Math. Monthly 94 (1987), 279-284.

Department of Information

The Shanghai University of Finance and Economics

Shanghai

China 